\title{
Pearls for Correct Assessment of Optic Disc at Glaucoma Diagnosis
}

\author{
Burak Turgut \\ Department of Ophthalmology, Faculty of Medicine, Yuksek Ihtisas University, Ankara, Turkey
}

DOl: https://doi.org/10.17925/USOR.2017.10.02.104

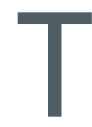

he correct evaluation of the optic disc, and related structures in ophthalmoscopy, is critical for the diagnosis of open angle glaucoma because usually glaucomatous optic nerve damage firstly occurs in the optic disc before detectable visual field defects become apparent; however, some studies have demonstrated that visual field defects can also be found without any glaucomatous changes in the optic disc. The purpose of this review is to provide a guide to clinicians for the evaluation of the optic disc in glaucoma suspects and patients. This guide can facilitate the diagnosis and exclusion of glaucoma in these subjects.

\section{Keywords}

Glaucoma, diagnosis, optic nerve head, optic disc, correct evaluation

Disclosure: Burak Turgut has nothing to declare in relation to this article. No funding was received in the publication of this article.

Compliance with Ethics: This study involves a review of the literature and did not involve any studies with human or animal subjects performed by any of the authors.

Authorship: All named authors meet the International Committee of Medical Journal Editors (ICMJE) criteria for authorship of this manuscript, take responsibility for the integrity of the work as a whole, and have given final approval to the version to be published.

open Access: This article is published under the Creative Commons Attribution Noncommercial License, which permits any non-commercial use, distribution, adaptation and reproduction provided the original author(s) and source are given appropriate credit.

Received: 21 April 2017

Accepted: 21 August 2017

Citation: US Ophthalmic Review, 2017;10(2):104-10

Corresponding Author: Burak Turgut, Yuksek Ihtisas University, Faculty of Medicine, Department of Ophthalmology, Street 1377, No.21, 06520, Ankara, Turkey.

E: drburakturgut@yahoo.com
The optic nerve head (ONH) is the region where retinal ganglion cells (RGCS) leave the eyeball via the scleral canal. It also consists of blood vessels, glial, and connective tissue. Glaucoma is a progressive optic neuropathy characterized by the loss of retinal nerve fibres and the axons of RGCs at the level of the $\mathrm{ONH}$. The primary site for glaucomatous damage is the $\mathrm{ONH} .{ }^{1-3}$ Although the $\mathrm{ONH}$ and the optic disc are often used to mean the same thing, in fact the optic disc is the part of the ONH which is observed in ophthalmoscopy. Although there are no clear lines demarcating the histologic layers of $\mathrm{ONH}$, the $\mathrm{ONH}$ consists of the surface retinal nerve fibre layer (RNFL), and pre-laminar, laminar (lamina cribrosa [LC]), and retrolaminar layers..$^{1-6}$ Therefore, we actually observe the superficial RNFL and the optic cup in the $\mathrm{ONH}$.

Glaucomatous optic nerve damage usually occurs in the ONH before there are any detectable visual field (VF) defects; however, some studies have demonstrated that VF defects can also be found without any glaucomatous changes in the optic disc..$^{7-9}$ It is understood that glaucomatous VF defects can be detected on standard automated perimetry when $20-40 \%$ of RGCs are lost. 10,11 Thus, the correct and careful evaluation of the optic disc and tissues immediately surrounding the disc are very important for detecting early glaucomatous damage. However, firstly it is crucial to know what the features and appearance of a healthy optic disc should be. In the examination of the $\mathrm{ONH}$, the size and shape of the optic disc should be correctly evaluated, as should:

- the size, color, and integrity of the neuroretinal rim (NRR);

- the size and shape of the optic cup;

- the cup-to-disc ratio (CDR);

- the shape and configuration of the vessels in the $\mathrm{ONH}$;

- the presence of the laminar dot sign in the cup;

- the changes in peripapillary region (ONH hemorrhages, peripapillary atrophy [PPA]); and

- the loss of lines belong to RNFL in red-free illumination (Figures $1 A$ and $B$, and $2 A-F$ ). ${ }^{1-6,12}$

The main techniques for clinical examination of the $\mathrm{ONH}$ include direct ophthalmoscopy, indirect ophthalmoscopy, and slit-lamp biomicroscopy with a contact lens or non-contact handheld lens $(+66,+78,+90$ diopter [D]) or a Hruby lens. The use of a slit lamp with a handheld lens is the preferred method because it provides good stereopsis and magnification for the physician and also convenience for the patient. ${ }^{13-19}$

Although retinal tomography and optical coherence tomography (OCT) are useful methods to perform some measurements such as optic disc diameter, CDR, NRR thickness and optic disc area, direct evaluation of the optic disc by the physician is necessary. 
Table 1: The correction factors commonly used in fundus lenses in the evaluation of the size of the optic nerve head ${ }^{12-22}$

\begin{tabular}{|l|l|}
\hline Condensing fundus lens & Correction factor \\
\hline Volk 60 D & 0.94 \\
\hline Volk 78 D & 1.13 \\
\hline Volk 90 D & 1.36 \\
\hline Volk Super 66 & 1.00 \\
\hline Volk Superfield & 1.50 \\
\hline Nikon 60 D & 1.03 \\
\hline Nikon 90 D & 1.59 \\
\hline
\end{tabular}

\section{Optic disc size, color, and shape}

The optic disc is the ophthalmoscopically visible part of the $\mathrm{ONH}$. The boundaries of the optic disc conform to the edges of the scleral canal, which appears as a whitish circular band. In the other words, the size of the scleral canal determines the optic disc size. Eyes with small scleral canals have small optic discs (high hyperopia) and eyes with large scleral canals have large discs (high myopia). Black subjects tend to have larger discs than white subjects. The borders of the optic disc are defined as the innermost border of reflective tissue that is internal to any pigmented tissue and within which only neuroretinal tissue is present. It can be difficult to determine the borders of the optic disc in individuals with high myopia and eyes with significant PPA. ${ }^{20-25}$

It is critical to first correctly estimate the size of the optic disc when investigating for glaucoma. Measurement of optic disc size is best performed at the slit lamp using a narrow vertical light beam and a condensing fundus lens. Depending on the fundus lens that is used, the size measured at the slit lamp should be multiplied by the correction factor. As a $60 \mathrm{D}$ lens has a correction factor of approximately $\mathrm{x} 1$, it is the optimal lens for the measurement of optic disc size. The correction factors commonly used in the evaluation of the size of the $\mathrm{ONH}$ are given in Table 1..$^{13,15,25} \mathrm{The} \mathrm{ONH}$ diameter can be calculated using the formula:

\section{ONH diameter $(\mathrm{mm})=(\mathrm{X} / \mathrm{H}) \times \mathrm{D} \times \mathrm{C}$}

$(X=$ height of beam $(\mathrm{mm}) ; \mathrm{H}=$ height setting on the beam height indicator $(\mathrm{mm}) ; \mathrm{D}=$ diameter of the optic disc measured by the beam height indicator (mm); $\mathrm{C}=$ correction factor).

Additionally, the area of the $\mathrm{ONH}$ can be calculated using a modified formula: $\mathrm{ONH}$ area $\left(\mathrm{mm}^{2}\right)$ : $\mathrm{r} / 4 \mathrm{X}$ horizontal disc diameter $\mathrm{X}$ vertical disc diameter $\left(r=\right.$ the correction factor based on used lens diopter). ${ }^{13,15,25}$

In the direct ophthalmoscope with two spot sizes, the small light spot, which is 5 degrees in size, is approximately the size of the average optic disc, whereas in those with three spot sizes, the middle spot may be used for the assessment of optic disc size. Firstly, the light spot is aligned over or next to the optic disc. Then, the size of the optic disc is compared with the spot size. If the disc is inside the illumination area of the light spot, it is a small disc. If the optic disc exceeds this spot area, this means that optic disc is large. ${ }^{15-19,25}$

The distance between the optic disc and the fovea as disc diameters can be used for the evaluation of the optic disc size. This distance as measured from the temporal edge of the optic disc to the center of the fovea is
Figure 1: The right (A) and the left (B) eyes of a case with healthy optic nerve head
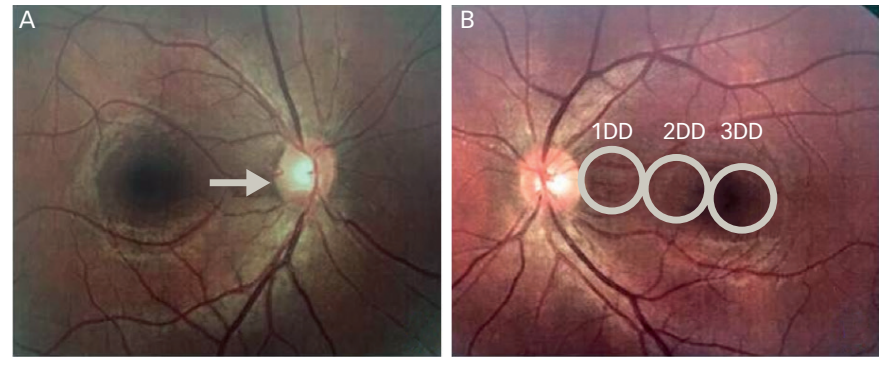

The white arrow indicates zone alpha $(\alpha)$. The circle corresponds with a normal optic disc size. The distance between the optic disc and foveola corresponds with two and a half optic disc diameter. $D D=$ disc diameter.

Figure 2: Glaucomatous optic nerve heads from various stages of glaucoma (A-D)
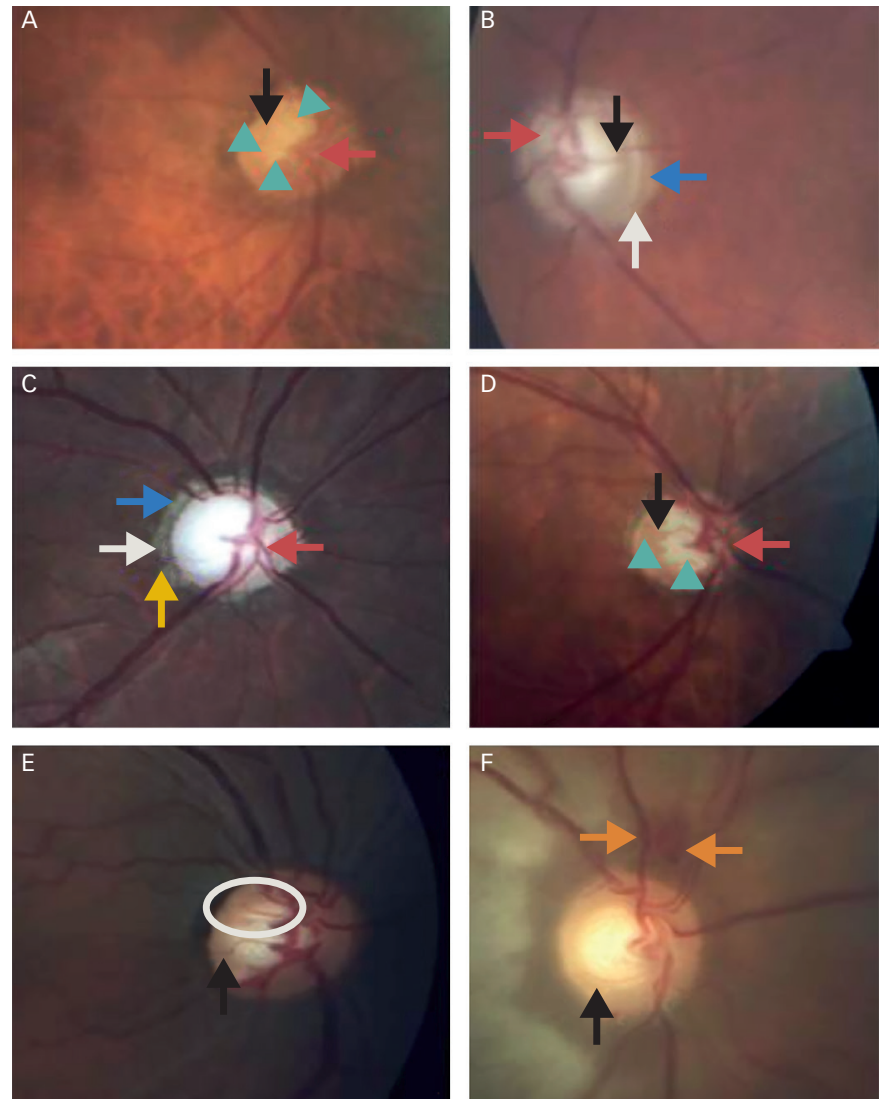

The blue arrows indicate zone alpha $(\alpha)$. The white arrows point out zone beta ( $\beta)$. Red arrows indicate nasalization. The yellow arrow indicates bean potting. Black arrows point out bayonetting. Green arrowheads indicate the area of the laminar dot sign. Orange surrounds the optic nerve head (ONH) hemorrhage. The circle indicates the baring of the circumlinear vessels in $\mathrm{ONH}$.

approximately two to three disc diameters in eyes with normal size and axial length. A shorter distance indicates a larger disc, and a longer one signifies a smaller optic disc (Figure 1B).20.21

In estimating optic disc size, slit lamps with an adjustable vertical light beam, a scale calibrated in millimetres and a magnifying fundoscopy lens should be used. Firstly, the coaxial slit beam is placed on the observation axis and adjusted to the vertical and horizontal diameters of the optic disc 
using the inner margin of Elschnig's white scleral ring. After at least three measurements are taken, the average is multiplied by the correction factor of the lens which was used..$^{13,15,25}$ Elschnig's scleral ring is a white circular band not belonging to the optic disc. It separates the intrapapillary region of the optic disc from the peripapillary area. If it is considered a part of the optic disc, the measurements of the larger NRR and smaller CDR are obtained. ${ }^{13-19}$

The average vertical optic disc diameter is $1.88 \mathrm{~mm}$ (vertically ranging from $1,700-2,000 \mu \mathrm{m}$ ), and the average horizontal diameter is $1.77 \mathrm{~mm}$ (ranging from 1,600-1,800 um). Thus, a normal healthy optic disc is usually vertically oval. A small vertical diameter is equal to or less than $1.5 \mathrm{~mm}$; a large vertical diameter is greater than $2.2 \mathrm{~mm} .^{1-6,12}$

The thickness of the central retinal vein (CRV) can also be used to help estimate optic disc size. The average thickness or diameter of CRV is approximately $125 \mu \mathrm{m}$ at the region which CRV crossed the inferior NRR in ONH hemorrhage (ONHH). If the optic disc has a width of about 12-14 CRV diameters, it can be considered that ONH is normal in size. A higher number of CRV diameters points out a larger $\mathrm{ONH}$, whereas a smaller number of CRV diameters refers a smaller $\mathrm{ONH} .{ }^{1-6,12}$

Optic disc surface area is between $2.1 \mathrm{~mm}^{2}$ and about $2.8 \mathrm{~mm}^{2}$ (the mean value of $2.69 \mathrm{~mm}^{2}$ ) with a range from $0.80 \mathrm{~mm}^{2}$ to $5.54 \mathrm{~mm}^{2}$ in an average Caucasian population without high myopia. Optic disc size varies from small to large according to racial heritage as follows: Caucasians, Hispanics, Asians, African-Americans. The subjects with a refractive error over $+5 \mathrm{D}$ have significantly smaller optic discs while those with a refractive error over -8 D have significantly larger optic discs compared with those with emmetropia. ${ }^{1-6,12,14-28}$

The large optic discs have large optic cups and apparently thin NRRs, even if the NRR area is larger in large discs. This may cause a large optic disc to seem glaucomatous. Contrarily, a small optic disc might hide the NRR thinning. Thus, a large cup in a large optic disc can be normal, while a small optic cup in small optic disc suggests glaucomatous optic neuropathy (GON). ${ }^{1-6,12,14-28}$

Pale cupping (pallor exceeding cupping) indicates a non-GON, such as anterior ischemic optic neuropathy (AION), compressional lesion to the optic nerve (tumor or hemorrhage), secondary optic atrophies due to papilledema, and inflammatory and infectious optic neuropathies. . $-6,12,14-28^{-28}$

Non-glaucomatous cupping can occur in AION, optic nerve compression (optic nerve tumour or hemorrhage), contusion, inflammation, and trauma of optic nerve, and hereditary optic neuropathies. In contrast to GON, in which the optic cup deepens and enlarges as associated with the glaucoma type and the level of intraocular pressure (IOP), the optic cup does not show significant enlargement in eyes with non-GON. ${ }^{1-6,12,21,25-31}$

Greenfield criteria consist of age less than 50 years, visual acuity less than 20/40, optic nerve pallor in excess of cupping, and vertically aligned VF defects can be used for the suspicious for non-glaucomatous cupping in patients with normal pressure. It has been recommended that patients who have one or more of the Greenfield criteria need to have neuroimaging performed to identify the causes of non-glaucomatous cupping. However, the absence of these criteria does not rule out the presence of nonglaucomatous cupping. ${ }^{28}$

\section{Optic cup and cup-to-disc ratio}

The optic cup is a central pale excavation in the $\mathrm{ONH}$. The optic cup includes glial tissue but not retinal nerve fibre or RGC axons. The pale color of the cup is due to the exposure of the collagenous LC and the loss of glial tissue. The area of pallor in the center of the disc frequently corresponds well with the area of the cup.

The evaluation of CDR in normal subjects, subjects with physiological cup, and glaucoma patients is difficult. Additionally, the application of the evaluation rules of CDR is not appropriate for subjects with an optic nerve or disc anomaly (tilted, hypoplasic, dysplasic nerves). The size of the optic cup always seems smaller in monoscopic examination than in stereoscopic examination. ${ }^{21,25-31}$

The direction or point of deviation of small blood vessels on the surface of the $\mathrm{ONH}$ is used to determine the size of the optic cup (contour method) but not the area of pallor in the center of the optic disc (colorcontrast method). ${ }^{21,25-31}$

Physiologically large cups can occur more commonly in patients with normally large chorioscleral canals and large discs. Optic cup size has high inter-individual variability. The area of the optic disc and cup are correlated in normal eyes. This means that large discs have larger cups and smaller discs have smaller cups. ${ }^{1-6,12}$ Thus, the optic disc area affects the CDR. A large cup in a large optic disc is most likely normal, while an average CDR in a small disc may be a sign of glaucomatous optic cupping. Optic cup depth depends on the cup area in normal eyes. This means that a larger cup has a deeper cup. In normal eyes, CDRs are larger horizontally than vertically. In normal eyes, the shape of the optic cup is horizontally oval as the vertical optic disc diameter is about $8 \%$ higher than the horizontal ones. ${ }^{1-6,12,15,21,25-31}$

The average CDR is 0.4. However, CDR ratio in normal patients can range from 0.0 to 0.9 because of inter-individual variation in disc size and cup size. Normal CDRs range from less than 0.3 ( $66 \%$ of normal individuals) to greater than 0.5 (only $6-10 \%$ of normal individuals). Large scleral openings cause large CDR while small openings give the appearance of a small CDR. ${ }^{1-6,12,21,25-31}$

A CDR less than 0.4 without an abnormally small optic disc size usually indicates a non-glaucomatous optic disc. A CDR between 0.4 and 0.8 which was calculated to the optic disc size may belong to a subject with a normal optic disc (physiologic cupping) or a glaucoma suspect or early/moderate glaucoma. A CDR equal or over 0.8 should be considered as a sign of GON unless proved otherwise. ${ }^{1-6,12}$

In the normal population, CDR is mostly symmetrical and the symmetrical increase in CDR is not common in glaucoma. More than CDR asymmetry, a difference of 0.2 between CDRs of both eyes, might be an early sign of glaucoma. ${ }^{1-6,12}$

Vertical elongation of the optic cup is due to the loss of RGC axons and the thinning of NRR in inferior and superior poles. The increase in CDR and the attenuation in NRR indicate diffuse RGC loss from GON.1-6,12,21,25-31 
Saucerization is a shallow diffuse excavation in the cup and it is very hard to detect in some cases because of the need for careful detection

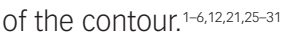

Estimation of CDR has only limited value in the identification of GON because of the wide variation in the size of the optic disc and cup in the normal population. In addition, there is the significant individual measurement variability among glaucoma specialists in the evaluation of CDR. ${ }^{29}$ In case of the adjustment for the optic disc size, CDR has a diagnostic value in early glaucoma.

\section{Neuroretinal rim}

NRR is the tissue in between the outer boundary of the cup and the optic disc margin. Normally, circumlinear blood vessels rest on the NRR. Therefore, in most cases, the boundaries of the optic cup are best identified by following the track and kink of the blood vessels within the ONH. PPA can cause the wrong determination of the boundary of the optic disc and the misinterpretation as NRR. ${ }^{1-6,12,21,30-35}$ To evaluate the width of the NRR thickness, the cup boundaries should be clearly identified.

The 'ISNT' rule is a helpful way to describe how the optic nerve is supposed to look in a healthy eye and states that in a healthy optic disc without a horizontally oval optical nerve, the NRR thickness from thick to thin is as Inferior, Superior, Nasal and Temporal, respectively. The ISNT rule gives to the optic cup a round or horizontally oval shape in healthy subjects. An NRR which is thinner and incompatible with the ISNT rule may be a sign of GON. . $-6,12,21,30-35^{-1}$

NRR color in normal healthy subjects should be pink or orange due to there being about 1-1.2 million RGC axons and capillaries without any localized thinning. The width of NRR is the distance between the border of the optic disc and the position of blood vessel bending. In glaucoma, NRR always seems pink except in terminal glaucoma. This means that NRR is pallor, it is not glaucoma. Pallor of the NRR increases the likelihood that a non-GON is present, especially when pallor is greater than cup size. Increasing pallor of the optic disc and especially of the NRR is a typical sign of optic nerve damage. A mis-match between pallor and NRR margin can be a sign of early glaucoma. ${ }^{1-6,12,21,30-35}$

NRR thinning is defined as generalized (diffuse) loss in NRR, while NRR notching defines the focal loss of NRR. Additionally, it has been demonstrated that NRR notching corresponds well with VF loss in most cases, although not all. The typical sequence of NRR loss in glaucoma is the infero-temporal and supero-temporal, followed by the temporal, infero-nasal, supero-nasal and, finally, the nasal sectors. ${ }^{1-6,12,21,230-35}$

NRR notching usually does not occur in the temporal or nasal region. Temporal NRR notching without other glaucomatous optic disc changes is most likely associated with an optic disc insertion anomaly. If NRR notching is present, this can indicate the glaucoma, even if CDR is the normal value. . $-6,12,21,30-35^{-}$

In early glaucoma, NRR thinning predominantly occurs in the inferotemporal and supero-temporal disc regions while in moderate glaucoma, it is observed in the temporal horizontal disc region. In advanced glaucoma, the remnants of NRR are present mainly in the nasal optic disc region. This sectorial sequence of the NRR thinning generally correlates with the progression of VF defects in glaucoma. ${ }^{1-6,12,21,20-35}$

\section{Optic nerve head hemorrhage}

$\mathrm{ONHH}$ is also known as RNFL hemorrhage, splinter hemorrhage, drance hemorrhage, or peripapillary hemorrhage, is a splinter or flame-shaped hemorrhage located on the disc margin or within 1 disc diameter of $\mathrm{ONH}$ and in the RNFL, but not in the cup (Figure 2F). ONHH likely occurs due to the infarction of the blood supply to the $\mathrm{ONH} \cdot{ }^{36-40}$

ONHH is observed rarely in normal eyes and appears more often in eyes with glaucoma. $\mathrm{ONHH}$ at the border of the optic disc is a highly pathognomonic finding of GON. It can be found in early and moderate stages of glaucoma and in normal tension glaucoma (NTG), primary open angle glaucoma (POAG) and ocular hypertension (OHT). However, it is more common in patients with NTG. ONHH is rare in advanced glaucomatous cupping likely due to absence of sufficient NRR tissue. ONHH associated with early glaucoma is usually located on the supero-temporal and inferotemporal regions of the optic disc. ${ }^{36-40}$

$\mathrm{ONHH}$ is also a strong indicator for the progression of glaucoma. The Early Manifest Glaucoma Trial (EMGT) and Collaborative NTG study have demonstrated that $\mathrm{ONHH}$ was strongly associated with progression. ${ }^{37,39}$ In addition, the EMGT showed that the reduction of IOP did not alter $\mathrm{ONHH}$ frequency. ${ }^{37}$ Additionally, it has been reported in the Ocular Hypertension Study (OHTS) that an ONHH in cases with OHT was associated with an increased risk of developing POAG; However, 87\% 0 $\mathrm{f}$ eyes in which ONHH was developed did not convert to POAG in a timespan of 96-months in this trial..$^{38}$

ONHH typically occurs where NRR notching or RNFL defect is observed. ONHH can also precede NRR notching, localized RNFL defect and VF loss. The color of $\mathrm{ONHH}$ depends upon how long it has been present. Although $\mathrm{ONHH}$ is transient, it can be recurrent and, in each recurrence, it typically occurs in the same region on the disc. ONHH often recurs in the same area of the disc until a notch is formed, and then occurs at other areas of the disc where the rim is still normal. ${ }^{36-40}$

$\mathrm{ONHH}$ is more common in patients with large IOP variations. ONHH typically resolves within 6-10 weeks of onset and is associated with localized RNFL defects and NRR notching. ONHH is usually missed in clinical observation because of its resolving time..$^{36-40}$

Non-glaucomatous $\mathrm{ONHH}$ can occur in patients with posterior vitreous detachment, branch retinal vein occlusion, diabetic retinopathy, hypertensive retinopathy, AION, and in patients with anaemia. ${ }^{36-40}$

\section{Laminar dot sign}

The The LC is the part of the sclera with a mesh-like structure and is the exit zone from the globe for the axons of RGCs or retinal nerve fibres. It has a lot of pores formed by the multi-layered network of collagen fibres. It has been considered that the $\mathrm{LC}$ is a structure which maintains the pressure gradient between IOP and the periocular pressure. ${ }^{1-5,41,42}$

The LC is very sensitive to the effects of the IOP because it is structurally weaker compared with the thicker and denser scleral parts. The elevation of IOP causes the posterior displacement or bulging of the $L C$, deformation of the pores and compression of the nerve fibres and blood vessels traversing though laminar pores. It is believed that posterior displacement is one of the causes of GON. ${ }^{1-5}$ 
The superior and inferior poles of LC in particular have less structural support for the axon bundles leaving the nerve because they have the largest pores. Thus, the damage to the RGC axons occurs typically as greater in the superior and inferior poles of the optic nerve..$^{1-6,12}$

Laminar dot sign is due to the exposure of the pores in LC or the increase in visibility of the LC pores because of the loss of RGC axons. Although laminar dot sign may be observed in healthy non-glaucomatous eyes and they are in the round form in these eyes, it is more common in eyes with GON than in normal eyes. It has been reported that the laminar dot sign was observed in almost $70 \%$ of patients with open-angle glaucoma and in only almost $30 \%$ of healthy subjects. Laminar pores in glaucomatous eyes are usually in the shape of a slit. It has been reported that the size and shape of LC pores may predict glaucomatous VF loss (Figure $2 A$ and $D$ ). ${ }^{1-5,41,42}$

\section{Parapapillary atrophy, zones alpha and beta}

PPA refers to the thinning and degeneration of the chorioretinal tissue just outside of the optic disc and the exposure of the retinal pigment epithelium (RPE), which gives the tissue a moth-eaten appearance. It is considered that PPA occurs due to ischemia in the peripapillary choroidal circulation and/ or vascular deficiency in the ONH. PPA differs from normal peripapillary choroidal crescents with high myopia and the inferior scleral crescent in eyes with tilted optic discs in the respect that PPA is typically irregular and patchy, whereas choroidal crescent is typically very uniform in color and shape. Additionally, in the myopic crescent, only the inner limiting membrane and underlying RNFL, or its remnants, cover the sclera. $5,15,21,25,43,44$

PPA may also be present in normal eyes; its frequency is higher in glaucomatous eyes with especially shallow optic cupping compared with normal eyes, or in eyes with OHT. If it is associated with glaucoma, PPA is typically located in the region adjacent to NRR thinning. ${ }^{25,43,44}$

PPA is divided into the peripheral zone alpha $(\alpha)$ and the central zone beta $(\beta)$. If both PPA zones are present, zone $\alpha$ is always peripheral to zone $\beta$, which is closer to the optic disc. It has been reported that although both zones were larger in glaucoma patients, only zone $\beta$ was more frequent in patients with glaucoma compared with healthy controls. $5,15,21,25,43,44$

Zone $\alpha$ is a peripapillary crescent region characterized by RPE irregularities including hypo and hyperpigmented areas and it is associated with thinning of the chorioretinal tissue layer (Figure $1 A$, Figure $2 B$ and $C$ ). Nasally, it is always separated from the neuro-retinal rim by either zone $\beta$, if present, or by the scleral ring if zone $\beta$ is absent, and temporally it is bounded by the normal retina. Zone $\alpha$ is non-specific finding because it is observed in most normal eyes and in some glaucomatous eyes. Additionally, it is more common than zone $\beta .5,15,21,25,43,44$

Zone $\beta$ is the irregular central zone immediately adjacent to the optic disc, characterized by visible underlying sclera and large choroidal vessels due to the complete RPE and choriocapillaris atrophy RPE atrophy. Zone $\beta$ is more common and extensive in glaucomatous eyes compared with healthy normal eyes and the eyes with $\mathrm{OHT}$, also it is associated with progression of glaucoma. A localized zone $\beta$ is correlated with focal VF defects and progression of VF loss and it may precede NRR notching, ODHH, and RNFL Ioss. It has been reported that zone $\alpha$ occurred in $85 \%$ of the normal eyes, while zone $\beta$ was observed in only $15 \%$ of the normal eyes. It has been suggested that the presence of zone $\beta$ in particular is associated with subsequent progression to glaucomatous damage. ${ }^{43,44}$

However, a study by Savatorsky et al. showed that parapapillary atrophy does not always correlate with glaucoma progression, and in this study neither zone $\alpha$ nor zone $\beta$ area correlated with progression in OHTS. ${ }^{46}$

\section{Evaluation of retinal nerve fibre layer and detection of retinal nerve fibre layer defects}

RNFL is composed of axons of RGCs, which are covered by astrocytes and bundled by Muller cell processes. The normal RNFL in a healthy eye is seen as bright or white fine striations radiating outwards from the optic disc. These striations are most prominent in infero-temporal and superotemporal quadrants. In these regions in which the RNFL is thickest, the retina has a glistening appearance. ${ }^{1-6,12}$

The normal RNFL can be visualized with ophthalmoscopy, OCT, or retinal tomography. The best method for this purpose is the ophthalmoscopic or slit-lamp biomicroscopic observation with a fundus lens, bright light, and red-free or green light filters. The green light is absorbed by the RPE and choroid and allows easier visualization of the RNFL in a dark background because the light is reflected by the RNFL. However, the magnification correction according to the lens that was used (a reduction to 6 or $10 x$ for a $78 \mathrm{D}$ or $90 \mathrm{D}$ lens) should be performed in the ophthalmoscopic RNFL evaluation. ${ }^{15,31-33}$

In the evaluation of RNFL, the brightness and striations of the RNFL and the visibility of the parapapillary vessels should be observed. Parapapillary vessels typically seem indistinct in a healthy eye because healthy RNFL will obscure the details of underlying peripapillary retinal vascular walls. ${ }^{15,31-33}$

RNFL defects appear as the loss of normal striations in the retina or darker zones in the areas of expected brightness belonging to healthy RNFL. If RNFL loss is developed, the walls of the peripapillary vessels have a clearer, redder and darker appearance and increased sharpness and visibility. It is well known that the earliest changes in GON are often presented with RNFL defects in the $\mathrm{ONH}$ and that glaucomatous VF loss follows the RNFL defects. In other words, RNFL defects are an important sign of pre-perimetric glaucoma. ${ }^{1-6,12}$

RNFL defects in glaucoma are caused by selective damage to the superior and inferior arcuate bundles, without the significant involvement of papillo-macular and nasal retinal nerve fibre bundles. RNFL defects are most visible in the temporal inferior sector, followed by the temporal superior, the nasal superior and the nasal inferior sector, correlated with the sectors' sequence with respect to rim configuration and retinal artery calibre. Localized RNFL defects are detected more easily and they are most often located in the temporal inferior sector followed by the temporal superior sector. They are rarely found in the nasal region. ${ }^{1-6,12,15,31-33}$

Although the defects can occur in the shape of a slit or a wedge, the wedge defects are more common than slit defects in glaucoma. Focal RNFL defect is usually associated with a notch at the disc, or with a current or prior ONHH that developed 6-8 weeks earlier. ${ }^{1-6,12,31-33}$

RNFL defects generally precede glaucomatous disc changes and VF loss. RNFL loss is $85 \%$ specific for glaucoma. RNFL defects follow an arcuate 
pattern, as would be expected from the normal RNFL anatomy. True RNFL defects are at least an arteriole in width and extend back to the optic disc compared with pseudo defects, which may be thin or may never extend to the optic nerve. ${ }^{1-6,12,15,31-33}$

Diffuse RNFL defects may be best identified by the comparison of the striations among the superior and inferior poles and the temporal and nasal regions in the same eye, as well as the striations between both eyes, and with the detection of general reduction in RNFL brightness, increased sharpness and prominence of peripapillary vessels, and clearer visualization of the underlying choroid. ${ }^{1-6,12,15,31-33}$

\section{Bean potting in the optic nerve head}

Bean potting, also described as 'shelving', occurs in late glaucoma. However, it can also present in a non-glaucomatous normal nerve. It is described as vessels visibly entering the optic cup over the NRR, disappearing behind the excavated tissue, and re-emerging at the bottom of the cup (Figure 2 C). ${ }^{45}$

\section{Baring of circumlinear vessels in the optic nerve head}

Normally, circumlinear blood vessels rest on NRR tissue within the optic disc. Baring of circumlinear vessels often occurs normally and it is not pathognomonic of glaucoma. Baring of circumlinear vessels occurs in areas in which NRR loss was developed. Thus, it has been considered that the baring of circumlinear blood vessels possibly represents especially the focal loss of NRR or NRR notching. It is clinically characterized as the unsupported appearance of 'suspending' or 'hanging in mid-air' of the vessels crossed over the optic cup due to the lack of adjacent support of NRR in direct contact (Figure 2E). ${ }^{1-6,12}$

\section{Nasalization of blood vessels in the optic nerve head}

Nasalization of the $\mathrm{ONH}$ vessels is described as one of the ophthalmoscopic signs of GON. However, it can easily be observed in cases of advanced glaucomatous cupping but not in early glaucoma. It is due to the only structural support in the nasal NRR and the severe loss of superior, inferior, and temporal NRR tissue (Figure 2A to D). However, as the blood vessels commonly enter and leave the eye along the nasal border of the cup, they will appear nasalized when the cup is large, either physiologically or as a result of glaucoma. ${ }^{1-6,12}$

\section{Retinal arteriolar attenuation or construction in the optic nerve head}

A focal constriction of retinal arteriole in $\mathrm{ONH}$ likely results from RGC loss and a reduced metabolic demand from an increasingly thinner NRR or the superficial retinal layers. It is usually seen after VF defect has occurred. Vessel diameter reduces with decreasing area of NRR, diminishing visibility of the RNFL and increasing VF defects. A focal constriction of retinal arteriole in $\mathrm{ONH}$ can be caused by any optic nerve damage such as nonarteritic AION and GON. It is a weak finding for GON.1-6,12

\section{Bayonetting sign in vessels at the optic nerve head}

Retinal vessels deviate from their normal course with a sharp angulation of $90^{\circ}$ or more due to focal notching and significant NRR loss, and travel along the bottom of the optic cup. They then re-emerge at the edge of the rim from the deeply excavated cup with a sharp angle over the lip of the optic cup to pass over the disc margin. This travel gives them an appearance of a ' $z$ ' or a 'double angulation' (Figure $2 A, B, D, E, F$ ). It is typically seen in the $\mathrm{ONH}$ with advanced cupping or with localized NRR notching. ${ }^{1-6,12}$

\section{The five rules for evaluation of the optic disc in glaucoma diagnosis}

In recent years, some authors have recommended the use of a systematic and simplified system for the evaluation of the optic disc in glaucoma diagnosis called 'the five rules', which was devised by Fingeret et al. ${ }^{32}$ The rules defined in this system are the observation of:

- the scleral Ring for detection of the borders and size of the optic disc;

- the detection of Rim size;

- the evaluation of the RNFL;

- the evaluation the Region of parapapillary atrophy; and,

- observation of Retinal and optic disc hemorrhages. ${ }^{32}$

\section{Hallmarks of correct evaluation of the optic nerve head in suspected glaucoma}

- Measurement of the vertical ONH size at the slit lamp via dilated pupils.

- Detection of the vertical and horizontal CDR and careful consideration of the association between optic disc size and optic cup size.

- Assessment of the integrity of the NRR (diffuse NRR thinning, NRR notching, and NRR pallor) and the review for the characteristic ISNT rule.

- Exploration the presence of zone $\beta$ or extensive zone $\alpha$.

- Assessment of ONH for the presence of the vascular changes: $\mathrm{ONHH}$, baring or nasalization of vessels, and arteriolar narrowing.

- Assessment of the peripapillary and retinal RNFL integrity and exploration of focal or diffuse RNFL defects with a red-free filter.

\section{Conclusion}

Although an increased cup size, laminar dot sign, nasalization, vertical enlargement of the optic cup, and asymmetry between two eyes greater than 0.2 in the absence of disc size asymmetry are also predictive findings of GON, they are not specific for glaucoma. The single evaluation of CDR is not enough for consideration of the optic disc regarding glaucoma because there is high inter-observer and intra-observer variability among the measurements of CDR of even trained glaucoma specialists. The presence and occurrence of NRR notching, localized RNFL loss, the loss of ISNT rule, bayonetting in $\mathrm{ONH}, \mathrm{ONHH}$, and PPA in the form of zone $\beta$ show associations with open angle glaucomas. So, the detection of a few of these findings in an eye with suspected glaucoma is critical for the diagnosis of GON. $\square$ 
1. Nicolela MT, Optic Nerve: Clinical Examination. In: Giaconi JA, Law SK, Coleman AL, Caprioli J (eds.), Pearls of Glaucoma Management, Berlin Heidelberg: Springer-Verlag, 2010, 15-21.

2. Marjanovic I, The Optic Nerve in Glaucoma. In: Tomas Kubena (ed.). The Mystery of Glaucoma, Available at: www.intechopen $\mathrm{com} /$ books/the-mystery-of-glaucoma/the-optic-nerve-inglaucoma (accessed 29 August 2017)

3. Susanna Jr R, Medeiros FA, Ophthalmoscopic aspects of the optic nerve in glaucoma: Normal optic disc. In: Susanna Jr R, Medeiros FA (eds.), The Optic Nerve in Glaucoma, Rio de Jenerio: Cultura Médica, 2006, 1-12.

4. Bourne RR, The optic nerve head in glaucoma, Community Eye Health, 2006:19:44-5.

5. Jonas JB, Budde WM, Diagnosis and Pathogenesis of Glaucomatous Optic Neuropathy: Morphological Aspects, Prog Retin Eye Res, 2000;19:1-40.

6. Hayreh SS, Ischemic Optic Neuropathies, Berlin Heidelberg: Springer-Verlag, 2011

7. Raza AS, Hood DC, Evaluation of the structure-function relationship in glaucoma using a novel method for estimating the number of retinal ganglion cells in the human retina, Invest ophthalmol Vis Sci, 2015;56:5548-56.

8. Hood DC, Kardon RH, A framework for comparing structural and functional measures of glaucomatous damage, Prog Retin Eye Res, 2007;26:688-710

9. Malik R, Swanson WH, Garway-Heath DF, 'Structure-function relationship' in glaucoma: past thinking and current concepts, Clin Exp Ophthalmol, 2012:40:369-80.

10. Quigley HA, Dunkelberger GR, Green WR, Retinal ganglion cell atrophy correlated with automated perimetry in human eyes with glaucoma, Am J Ophthalmol, 1989;107:453-64.

11. Kerrigan-Baumrind LA, Quigley HA, Pease ME, et al., Number of ganglion cells in glaucoma eyes compared with threshold visual field tests in the same persons, Invest Ophthalmol Vis Sci, 2000;41:741-8

12. Kampougeris G, Spyropoulos D, Mitropoulou A, et al., Peripapillary retinal nerve fibre layer thickness measurement with SD-OCT in normal and glaucomatous eyes: distribution and correlation with age, Int J Ophthalmol, 2013;6:662-5.

13. Lim CS, O'Brien C, Bolton NM, A simple clinical method to measure the optic disk size in glaucoma, J Glaucoma $1996 ; 5: 241-5$.

14. Jonas JB, Optic disk size correlated with refractive error, Am J Ophthalmol, 2005:139:346-8.

15. Lachkar Y, Sellem E, [La papille glaucomateuse en pratique]
France: Chauvin B\&L, 2004. Available at: www.bausch-chauvin. com/tele/fichiers/33/Papille\%20glaucomateuse.pdf (accessed at September 22, 2017)

16. Siamak Ansari-Shahrezaei S, Maar N, Biowski R, Stur M, Biomicroscopic Measurement of the Optic Disc with a HighPower Positive Lens, Invest Ophthalmol Vis Sci, 2001:42:153-7.

17. Spencer $A F$, Vernon $S A$, Optic disc measurement: A comparison of indirect ophthalmoscopic methods, Br J Ophthalmol, 1995;79:910-5

18. Ansari-Shahrezaei S, Stur M, Magnification characteristic of a +90-diopter double-aspheric fundus examination lens, Invest Ophthalmol Vis Sci, 2002:43:1817-9.

19. Martínez de la Casa JM, Saenz Francés F, Fández Vidal AM, et al, Agreement between slit lamp examination and optical coherence tomography in estimating cup disc ratios, Eur J Ophthalmol, 2008;18:423-8

20. Grigera D, Clinical estimation of optic disc size, Glaucoma Now 2011; 1. Available at: https://pdfs.semanticscholar.org/e5f3/ a7b39fa5083eb5e3508247662c4e5bb00923.pdf (accessed 29 August 2017).

21. Jonas JB, Gusek GC, Naumann GO, Optic disc, cup and neuroretinal rim size configuration and correlations in norma eyes, Invest Ophthalmol Vis Sci, 1988;29:1151-8.

22. Broadway DC, Nicolela MT, Drance SM, Optic disc appearances in primary open-angle glaucoma, Surv Ophthalmol, 1999;43:S22343

23. Hoffmann EM, Zangwill LM, Crowston JG, Weinreb RN, Optic disk size and glaucoma, surv Ophthalmol, 2007;52:32-49.

24. Jonas RA, Wang $Y X$, Yang $H$, et al., Optic disc - fovea distance, axial length and parapapillary zones. The Beijing Eye Study 2011, PLOS ONE, 2015:10:e0138701.

25. Bhartiya S, Gadia R, Sethi HS, Panda A, Clinical evaluation of optic nerve head in glaucoma, J Curr Glaucoma Pract, 2010;4:115-32.

26. Gandhi M, Dubey S, Evaluation of the optic nerve head in glaucoma, J Curr Glaucoma Pract, 2013;7:106-14.

27. Reis ASC, Toren A, Nicolela MT, Clinical optic disc evaluation in glaucoma, European Ophthalmic Review, 2012;6:92-7.

28. Greenfield DS, Siatkowski RM, Glaser IS, et al., The cupped disc. Who needs neuroimaging?, Ophthalmology, 1998:105:186674.

29. Tielsch JM, Katz J, Quigley HA, Intraobserver and interobserver agreement in measurement of optic disc characteristics, Ophthalmology, 1988;95:350-6.

30. Spaeth GL, Lopes JF, Junk AK, et al., Systems for staging the amount of optic nerve damage in glaucoma: A critical review and new material, Surv Ophthalmol, 2006; 51:293-315.
31. Mackenzie PJ, Mikelberg FS, Evaluating optic nerve damage: pearls and pitfalls, Open Ophthalmol J, 2009;3:54-8.

32. Fingeret M, Medeiros FA, Susanna Jr R, Weinreb RN, Five rules to evaluate the optic disc and retinal nerve fiber layer for glaucoma, Optometry, 2005;76:661-8.

33. Uhm KB, Lee DY, Lee JS, Hong C, Sensitivity and specificity of qualitative signs to detect glaucomatous optic nerve damage, J Korean Ophthalmol Soc, 1998;39:153-62.

34. Lee JLS, Nicolela MT, Chauhan BC, Rates of neuroretinal rim and peripapillary atrophy area change, Ophthalmology, 2009;116:840-

35. Vingrys AJ, The many faces of glaucomatous optic neuropathy, Clin Exp Optom, 2000:83:145-60.

36. Siegner SW, Netland PA Optic disc hemorrhages and progression of glaucoma, Ophthalmology, 1996;103:1014-24.

37. Bengtsson B, Leske MC, Yang Z, Heijl A, EMGT Group, Disc hemorrhages and treatment in the early manifest glaucoma trial, Ophthalmology, 2008;115:2044-8

38. Budenz DL, Anderson DR, Feuer WJ, et al., Ocular Hypertension Treatment Study Group, Detection and prognostic significance of optic disc hemorrhages during the Ocular Hypertension Treatment Study, Ophthalmology, 2006;113:2137-43.

39. Drance S, Anderson DR, Schulzer M, Collaborative Normal-Tension Glaucoma Study Group, Risk factors for progression of visual field abnormalities in normal-tension glaucoma, Am J Ophthalmol, 2001;131:699-708.

40. Airaksinen PJ, Mustonen E, Alanku HI, Optic disk haemorrhages precede retinal nerve fibre layer defects in ocular hypertension, Acta Ophthalmol, 1981;59:627-41

41. Healey PR, Mitchell P, Visibility of lamina cribrosa pores and openangle glaucoma, Am J Ophthalmol, 2004;138:871-2.

42. Abe RY, Gracitelli CPB, Diniz-Filho A, et al., Lamina Cribrosa in Glaucoma: Diagnosis and Monitoring, Curr Ophthalmol Rep, 2015;3:74-84

43. Tezel G, Kolker AE, Kass MA, et al., Parapapillary chorioretina atrophy in patients with ocular hypertension. I. An evaluation as a predictive factor for the development of glaucomatous damage, Arch Ophthalmol, 1997:115:1503-8.

44. Tezel G, Kolker AE, Wax MB, et al., Parapapillary chorioretinal atrophy in patients with ocular hypertension. II. An evaluation of progressive changes, Arch Ophthalmol, 1997;115:1509-14.

45. Hitchings RA, Spaeth GL, The optic disc in glaucoma. I: Classification. Br J Ophthalmol, 1976;60:778-85.

46. Savatorsky E, Mwanza J-C, Budenz DL, et al., Longitudinal changes in peripapillary atrophy in the ocular hypertension treatment study, Ophthalmology, 2015;122:79-86. 